\title{
Second Harmonic Optical Vortex Generation in Air
}

\author{
Martynas Beresna and Peter G. Kazansky \\ Optoelectronics Research Centre, University of Southampton, SO17 1BJ, United Kingdom \\ pgk@orc.soton.ac.uk \\ Yuri Svirko \\ Department of Physics and Mathematics, University of Joensuu, FI-80101, Finland \\ Martynas Barkauskas and Romas Danielius \\ Light Conversion Ltd, LT-10223, Vilnius, Lithuania
}

\begin{abstract}
We demonstrate second harmonic vortex generation in atmospheric pressure air using tightly focused femtosecond laser beam. The average second harmonic power is two orders of magnitude higher compared to previous reports.

(C)2010 Optical Society of America

OCIS codes: (190.0190) Nonlinear optics; (260.7120) Ultrafast phenomena; (350.5400) Plasmas
\end{abstract}

High intensity ultrashort laser pulses have made it possible to discover such new phenomena as coherent X-ray generation, nonlinear Thomson scattering and frequency doubling in gases. In the experiments for the usually forbidden second harmonic generation (SHG) in low-pressure gases and metal vapors [1], low numerical aperture (NA) optics was conventionally used, Here we demonstrate second harmonic optical vortex generation in atmospheric pressure air using tightly focused femtosecond laser beam with linear and circular polarizations. The estimated conversion efficiency value was $4 \times 10^{-6}$, corresponding to two orders of magnitude increase in normalized efficiency and average second harmonic ( $\mathrm{SH}$ ) power compared to previously reported [2].

Experiments were performed with Yb:KGW based femtosecond system (Pharos, Light Conversion Ltd.) that delivered pulses of $270 \mathrm{fs}$ with repetition rate of $200 \mathrm{kHz}$ and wavelength centered at $1025 \mathrm{~nm}$. The power delivered to the focus was varied from $0.15 \mathrm{~W}$ to $2.5 \mathrm{~W}$. Consequently, the maximum radiation intensity in the focal spot was $10^{15} \mathrm{~W} / \mathrm{cm}^{2}$ (i.e. it was above the air ionization threshold of about $\sim 10^{14} \mathrm{~W} / \mathrm{cm}^{2}$ ). SHG was studied with linearly and circularly polarized pump beam, focused by x100/0.7 NA objective in ambient air.

Once the laser beam was focused with the objective small bluish spherical spot appeared in the focus with coherent SH radiation emerging from it (Fig. 1). The shape of SH pattern depended on the fundamental beam polarization. The linearly polarized fundamental beam produced SH radiation in form of two lobes. The lobes were oriented along the polarization azimuth and with electric field phase shifted by $\pi$ with respect to one another. The circular polarized fundamental beam produced the SH radiation, which was co-circular polarized and formed a ring around the beam axis.

Demonstrated results are explained as follows. SH could be generated in isotropic gaseous medium if the plasma in the irradiated volume is inhomogeneous [3]. The pondermotive force, which is electrons pushing from the center of the focused beam can induce such inhomogeneity and give rise to the SHG.
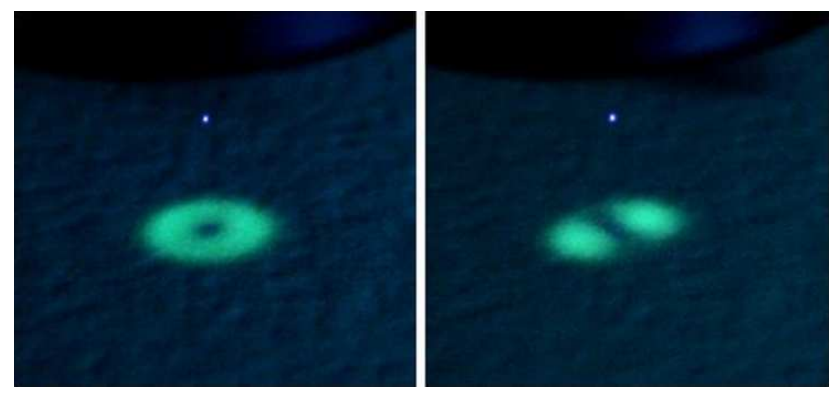

Fig. 1. Images of second harmonic pattern for circular (Left) and linear (Right) polarizations. On top of the photography the objective's edge can be seen.

Another interesting observation was a significant (about 3 times) enhancement of the SH signal accompanied by the plasma spot diameter increase in the presence of a weak nitrogen (Fig. 2) or compressed air flow at the focal area. Interestingly, the pressure needed for the maximum increase of the signal was bellow 0.1 bar, which is the lowest pressure measurable with our set-up. Yet higher gas pressure had produced merely several percent additional increase in the SH signal. Even stronger effect was noticed with compressed hydrofluorocarbon (HFC) gas flow from air duster. Blowing of this gas at the focus resulted in the up to twenty-fold increase of the SH signal. 
Consequently, the average power of signal was as high as $10 \mu \mathrm{W}$. The external gas flow drags positive ions out of the focus weakening plasma electric field strength and leading to stronger electron concentration gradient and thus to the enhanced SH emission.

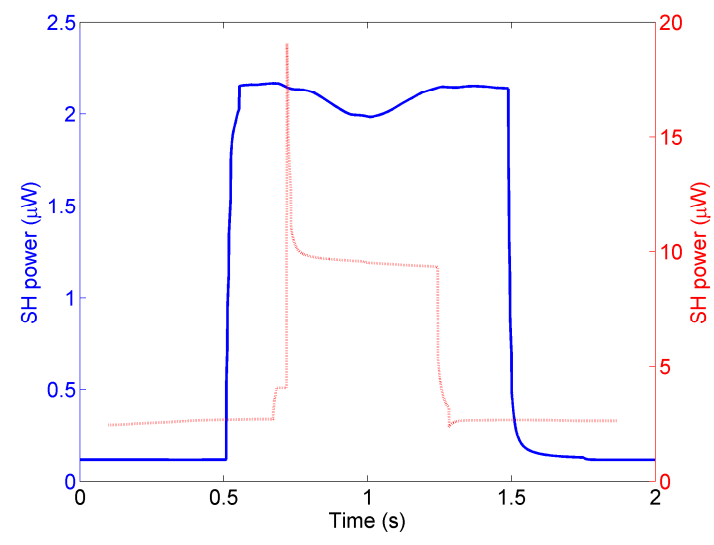

Fig. 2. Second harmonic power change when compressed HFC gas is blown at the focus spot for two pump powers. The signal increases about 20 times at $0.65 \mathrm{~W}$ pump power (continuous blue) and about 4 times at $2.45 \mathrm{~W}$ (dotted red).

For the circular polarization in the SHG process, a pair of the photons of the fundamental beam with angular momentum $2 \hbar$ creates only one SH photon with angular momentum $\hbar$ (Fig. 3 ). Thus the remaining angular moment should be either transferred to the plasma in the focal area or to the orbital angular momentum of the $\mathrm{SH}$ beam creating an optical vortex. The ring shaped pattern (typical for Laguerre-Gaussian $\mathrm{TEM}_{01 *}$ mode) observed in the far-field suggests presence of phase singularity and thus optical vortex generation.
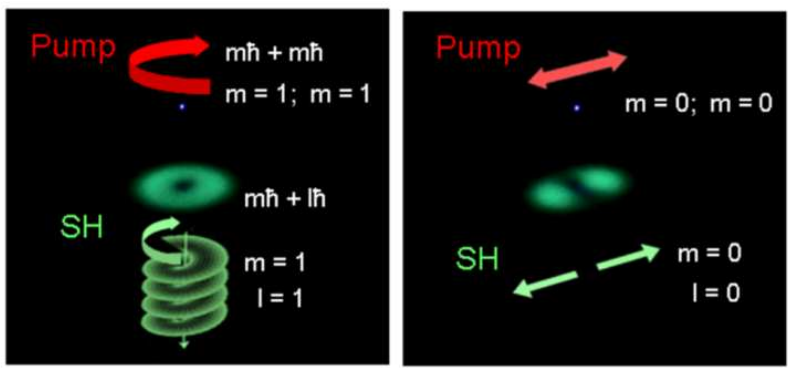

Fig. 3. Second harmonic vortex generated with circularly polarized pump in accordance with angular momentum conservation (Left). Two-lobe SH pattern with $\pi$ shifted electric fields for linearly polarized pump (Right).

Estimated effective second order nonlinearity in our experimental conditions is $0.03 \mathrm{pm} / \mathrm{V}$, which is of the same order as in poled silica fibres [4,5]. Increasing the interaction length to $1 \mathrm{~mm}$, e.g. using specially designed capillary or photonic crystal fiber efficiencies in access of $50 \%$ could be achieved.

In conclusion, we demonstrated high average power $(\sim 10 \mu \mathrm{W})$ SHG in ambient air with subpicosecond pulses at high repetition rate using tight focusing conditions. The efficient generation of circularly polarized SH vortex in air and the technique of efficiency increase via gas blow could find applications in optical trapping and manipulation.

\section{References}

[1] D. S. Bethune, "Optical second-harmonic generation in atomic vapors with focused beams,” Phys. Rev. A 23, 3139-3151 (1981).

[2] Y.-D. Qin, H.Yang, C.-J.Zhu, and Q.Gong, "Intense femtosecond laser-induced second-harmonic generation in atmospheric-pressure air," Appl. Phys. B 71, 581-584 (2000).

[3] Y. R. Shen, The principles of nonlinear optics (Wiley, New York, 1984).

[4] W. Margulis, F. Laurell, and B. Leschel, "Imaging the nonlinear grating in frequency-doubling fibres," Nature 378, 699-701 (1995).

[5] P. G. Kazansky and V. Pruneri, "Electrically stimulated light-induced second-harmonic generation in glass: evidence of coherent photoconductivity," Phys. Rev. Lett. 14, 2956-2959 (1997). 